\title{
A Facile and mild method for the synthesis of terminal bromofluoroolefins via diethylzinc promoted Wittig reaction.
}

\author{
Xinsheng LEI, Guillaume DUTHEUIL, Xavier PANNECOUCKE* and Jean-Charles \\ QUIRION
}

INSA de ROUEN, IRCOF, UMR CNRS6014, 1 rue Tesnière, 76131 Mont Saint-Aignan, France. Fax: (33) 2.35.52.29.59; Tel: (33) 2.35.52.24.27.

xavier.pannecoucke@insa-rouen.fr

\section{General Procedure for the obtention of bromofluoroolefins from aldehydes:}

To a solution of triphenylphosphine ( $2.4 \mathrm{mmol}, 1.2 \mathrm{eq}$ ), tribromofluoromethane $(2.4 \mathrm{mmol}$, 1.2 eq.), and an appropriate aldehyde ( $2.0 \mathrm{mmol}, 1.0$ eq.) in anhydrous THF (30-40 $\mathrm{mL})$, a solution of diethylzinc in hexanes or toluene $(2.4 \mathrm{mmol}, 1.2$ eq.) was added dropwise via a syringe pump over $30 \mathrm{~min}$. at room temperature under argon. The mixture was stirred at room temperature for 30 minutes. The resulting solution was then quenched with methanol $(10 \mathrm{ml})$, stirred for $20 \mathrm{~min}$, and concentrated under reduced pressure. The residue was then chromatographied on silica gel (eluent: cyclohexane/ ethyl acetate), affording desired bromofluoroolefins.

\section{General Procedure for the obtention of bromofluoroolefins from ketones:}

To a solution of triphenylphosphine $(3.0 \mathrm{mmol}, 1.5 \mathrm{eq}$.), tribromofluoromethane $(3.0 \mathrm{mmol}$, 1.5 eq.), and an appropriate ketone (2.0 mmol, 1.0 eq.) in anhydrous THF (30-40 mL ), a solution of diethylzinc in hexanes or toluene $(3.0 \mathrm{mmol}, 1.5$ eq.) was added dropwise via a syringe pump over $30 \mathrm{~min}$. at room temperature under argon. The mixture was stirred at room temperature for 30 minutes. The resulting solution was then quenched with methanol $(10 \mathrm{ml})$, stirred for 20 min., and concentrated under reduced pressure. The residue was then chromatographied on silica gel (eluent: cyclohexane/ ethyl acetate), affording desired bromofluoroolefins.

\section{Experimental Section:}

All commercial solvents were distilled before using. Tetrahydrofuran (THF) was distilled from sodium benzophenone ketyl under nitrogen atmosphere. TLC was performed on Merck 60F-250 silica gel plates and column chromatography over silica gel SI 60 (230-240 mesh). Flash column chromatography purifications were carried out using silica gel (70-230 mesh). ${ }^{1} \mathrm{H}$ NMR, ${ }^{13} \mathrm{C}$ NMR and ${ }^{19} \mathrm{~F}$ NMR $\left(\mathrm{CFCl}_{3}\right.$ as external reference) were recorded at 300.13 , 75.47 and $282.40 \mathrm{MHz}$ respectively . 


\section{Supplementary materials}<smiles>FC(Br)=CCCc1ccccc1</smiles>

${ }^{1} \mathrm{H}$ NMR $\left(300 \mathrm{MHz}, \mathrm{CDCl}_{3}\right)$ :

7.3-7.1 (m,5H), 5.6-5.4 (dt, $\left.0.5 \mathrm{H},{ }^{3} \mathrm{~J}=7.6 \mathrm{~Hz},{ }^{3} \mathrm{~J}_{\mathrm{H}-\mathrm{F}}^{\mathrm{cis}}=12.8 \mathrm{~Hz}\right), 5.1-4.9\left(\mathrm{dt}, 0.5 \mathrm{H},{ }^{3} \mathrm{~J}=7.6 \mathrm{~Hz}\right.$, $\left.{ }^{3}{ }^{\text {trans }}{ }_{\mathrm{H}-\mathrm{F}}=31.0 \mathrm{~Hz}\right), 2.7-2.6(\mathrm{~m}, 2 \mathrm{H}), 2.4-2.2(\mathrm{~m}, 2 \mathrm{H})$.

${ }^{19} \mathrm{~F}$ NMR $\left(282.5 \mathrm{MHz}, \mathrm{CDCl}_{3}\right)$ :

$-71.7\left(\mathrm{~d},{ }^{3} \mathrm{~J}_{\mathrm{H}-\mathrm{F}}^{\mathrm{cis}}=13.1 \mathrm{~Hz}\right), 75.4\left(\mathrm{dt},{ }^{3} \mathrm{~J}_{\mathrm{H}-\mathrm{F}}^{\text {trans }}=31.2 \mathrm{~Hz},{ }^{4} \mathrm{~J}_{\mathrm{H}-\mathrm{F}}=2.6 \mathrm{~Hz}\right)$

${ }^{13} \mathrm{C} \mathrm{NMR}\left(75.5 \mathrm{MHz}, \mathrm{CDCl}_{3}\right)$ :

139.5 (C5), 136.5-132.4 (d, $\left.{ }^{3} \mathrm{~J}_{\mathrm{C}-\mathrm{F}}^{\mathrm{cis}}=314.5 \mathrm{~Hz}, \mathrm{C} 1\right), 132.7-128.5\left(\mathrm{~d},{ }^{3} \mathrm{~J}^{\text {transs }}{ }_{\mathrm{C}-\mathrm{F}}=319.7 \mathrm{~Hz}, \mathrm{C} 1\right)$, 127.1-127.2-127.3 (C6, C7), 125 (C8), 110.5 (d, J $\left.{ }_{\mathrm{C}-\mathrm{F}}^{\mathrm{cis}}=12.7 \mathrm{~Hz}, \mathrm{C} 2\right), 107.9$ (d, J ${ }^{\text {trans }}{ }_{\mathrm{C}-}$ $\mathrm{F}=15.5 \mathrm{~Hz}, \mathrm{C} 2), 33.8\left(\mathrm{~d}, \mathrm{~J}_{\mathrm{C}-\mathrm{F}}^{\text {trans }}=2.3 \mathrm{~Hz}, \mathrm{C} 4\right), 33.5\left(\mathrm{~d}, \mathrm{~J}_{\mathrm{C}-\mathrm{F}}^{\text {trans }}=2.3 \mathrm{~Hz}, \mathrm{C} 4\right), 28.2(\mathrm{C} 3, \mathrm{cis}), 28.1$ $(\mathrm{C} 3$, trans)

MS (EI):230 (M+), $149\left(\mathrm{M}^{+}-\mathrm{Br}\right), 91$

IR (KBr): 3320, 3028, 2927, 1807, 1718, 1667, 1603, 1495, 1454, 1178, 1111, 1088, 1022, $1000,747,698$

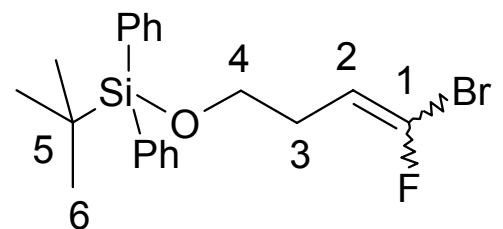

${ }^{1} \mathrm{H}$ NMR $\left(300 \mathrm{MHz}, \mathrm{CDCl}_{3}\right)$ :

7.7-7.6 (m, 4H, Ar-H), 7.5-7.3 (m, 6H, Ar-H), 5.7-5.5 (dt, 0.5H, ${ }^{3} \mathrm{~J}_{\mathrm{H}-\mathrm{F}}^{\mathrm{cis}}=13.3 \mathrm{~Hz},{ }^{3} \mathrm{~J}_{\mathrm{H}-\mathrm{H}}=7.8 \mathrm{~Hz}$, $\mathrm{H} 2), 5.2-5.0\left(\mathrm{dt}, 0.5 \mathrm{H},{ }^{3} \mathrm{~J}_{\mathrm{H}-\mathrm{F}}^{\text {trans }}=31.2 \mathrm{~Hz},{ }^{3} \mathrm{~J}_{\mathrm{H}-\mathrm{H}}=7.8 \mathrm{~Hz}, \mathrm{H} 2\right), 3.7-3.6(\mathrm{~m}, 2 \mathrm{H}, \mathrm{H} 4), 2.4-2.2(\mathrm{~m}$, 2H, H3), 1.1-1.0 (s, 9H, H6)

${ }^{19} \mathrm{~F}$ NMR $\left(282.5 \mathrm{MHz}, \mathrm{CDCl}_{3}\right)$ :

$70.6\left(\mathrm{~d},{ }^{3} \mathrm{~J}_{\mathrm{H}-\mathrm{F}}^{\mathrm{cis}}=13.2 \mathrm{~Hz}\right), 75.0\left(\mathrm{dt},{ }^{3} \mathrm{~J}_{\mathrm{H}-\mathrm{F}}^{\text {trans }}=31.2 \mathrm{~Hz},{ }^{4} \mathrm{~J}_{\mathrm{H}-\mathrm{F}}^{\text {trans }}=2.1 \mathrm{~Hz}\right)$

${ }^{13} \mathrm{C}$ NMR (75.5 MHz, $\left.\mathrm{CDCl}_{3}\right)$ :

138.5-134.9 (d, $\left.{ }^{1} \mathrm{~J}_{\mathrm{C}-\mathrm{F}}^{\mathrm{E}}=277 \mathrm{~Hz}, \mathrm{C}_{1}\right), 134.3-130.6\left(\mathrm{~d},{ }^{1} \mathrm{~J}_{\mathrm{C}-\mathrm{F}}^{\mathrm{Z}}=255 \mathrm{~Hz}\right), 136.0-130.0-128.1\left(\mathrm{C}_{\mathrm{Ar}} \mathrm{H}\right)$, $134.0\left(\mathrm{C}_{\mathrm{Ar}}\right), 110.0\left(\mathrm{~d},{ }^{2} \mathrm{~J}_{\mathrm{C}-\mathrm{F}}^{\mathrm{F}}=12.7 \mathrm{~Hz}, \mathrm{C}_{2}\right), 107.0\left(\mathrm{~d},{ }^{2} \mathrm{~J}_{\mathrm{C}-\mathrm{F}}^{\mathrm{E}}=277 \mathrm{~Hz}, \mathrm{C}_{2}\right), 62.0\left(\mathrm{dd},{ }^{4} \mathrm{~J}_{\mathrm{C}-\mathrm{F}}=2.1 \mathrm{~Hz}\right.$, $\left.{ }^{4} \mathrm{~J}_{\mathrm{C}-\mathrm{F}}=2.1 \mathrm{~Hz}, \mathrm{C}_{4}\right), 31.5\left(\mathrm{~d},{ }^{3} \mathrm{~J}_{\mathrm{C}-\mathrm{F}}^{\mathrm{Z}}=3.5 \mathrm{~Hz}, \mathrm{C}_{3}\right), 30.0\left(\mathrm{~d},{ }^{3} \mathrm{~J}^{\mathrm{E}}{ }_{\mathrm{C}-\mathrm{F}}=2.1 \mathrm{~Hz}, \mathrm{C}_{3}\right), 27.0\left(\mathrm{C}_{6}\right), 19.6\left(\mathrm{C}_{5}\right)$.

MS (EI): 351, $349\left(\mathrm{M}^{+}-\mathrm{C}_{4} \mathrm{H}_{9}\right)$ 
<smiles>[3H]C(/C=C(/[14CH3])F)CO[Si](c1ccccc1)(c1ccccc1)C(C)(C)C</smiles>

${ }^{1} \mathrm{H}$ NMR $\left(300 \mathrm{MHz}, \mathrm{CDCl}_{3}\right)$ :

7.8-7.6 (m, 4H, ArH), 7.6-7.4 (m, 6H, ArH), 5.5-5.4 (dd, ${ }^{3} \mathrm{~J}_{\mathrm{H}-\mathrm{H}}=9.7 \mathrm{~Hz},{ }^{3} \mathrm{~J}^{\mathrm{cis}}{ }_{\mathrm{H}-\mathrm{F}}=13.3 \mathrm{~Hz}, 0.5 \mathrm{H}$, $\mathrm{H} 2), 5.1-4.9\left(\mathrm{dd},{ }^{3} \mathrm{~J}_{\mathrm{H}-\mathrm{H}}=9.7 \mathrm{~Hz},{ }^{3}{ }^{\text {trans }}{ }_{\mathrm{H}-\mathrm{F}}=31.7 \mathrm{~Hz}, 0.5 \mathrm{H}, \mathrm{H} 2\right), 3.7-3.5(\mathrm{~m}, 2 \mathrm{H}, \mathrm{H} 4), 3.0-2.8(\mathrm{~m}$, 0.5H, H3trans), 2.7-2.5 (m, 0.5H, H3cis), 1.2 (s, 9H, H6), 1.2-1.1 (m, 3H, H7)

${ }^{19} \mathrm{~F}$ NMR (282.5 $\left.\mathrm{MHz}, \mathrm{CDCl}_{3}\right)$ :

$-71.7\left(\mathrm{~d},{ }^{3} \mathrm{~J}_{\mathrm{H}-\mathrm{F}}^{\mathrm{cis}}=13.3 \mathrm{~Hz}\right),-74.7\left(\mathrm{~d},{ }^{3} \mathrm{~J}_{\mathrm{H}-\mathrm{F}}^{\text {trans }}=31.7 \mathrm{~Hz}\right)$

${ }^{13} \mathrm{C}$ NMR (75.5 MHz, $\left.\mathrm{CDCl}_{3}\right)$ :

137.6-133.5 (d, $\left.{ }^{1} \mathrm{~J}_{\mathrm{C}-\mathrm{F}}^{\mathrm{cis}}=315 \mathrm{~Hz}, \mathrm{C}_{1}\right), 136.1-133.5-128.1\left(\mathrm{C}_{\mathrm{Ar}} \mathrm{H}\right), 134.0$ (dd, $\mathrm{C}_{\mathrm{Ar}}$, cis, trans), 134.3-130.1 ( $\left.{ }^{1} \mathrm{~J}_{\mathrm{C}-\mathrm{F}}^{\text {trans }}=315.7 \mathrm{~Hz}, \mathrm{C}_{1}\right), 116.0\left(\mathrm{~d},{ }^{2} \mathrm{~J}_{\mathrm{C}-\mathrm{F}}^{\mathrm{cis}}=12.1 \mathrm{~Hz}, \mathrm{C}_{2}\right), 113.3\left(\mathrm{~d},{ }^{2} \mathrm{~J}^{\text {trans }}{ }_{\mathrm{C}-\mathrm{F}}=13.8 \mathrm{~Hz}\right.$, $\left.\mathrm{C}_{2}\right), 68.0\left(\left(\mathrm{~d},{ }^{4}{ }^{\mathrm{trans}}{ }_{\mathrm{C}-\mathrm{F}}=2.3 \mathrm{~Hz}, \mathrm{C}_{4}\right), 67.8\left(\mathrm{~d},{ }^{4} \mathrm{~J}_{\mathrm{C}-\mathrm{F}}^{\mathrm{cis}}=2.9 \mathrm{~Hz}, \mathrm{C}_{4}\right), 36.7\left(\mathrm{~d},{ }^{3} \mathrm{~J}^{\mathrm{cis}}{ }_{\mathrm{C}-\mathrm{F}}=3.5 \mathrm{~Hz}, \mathrm{C}_{3}\right)\right.$, 34.8 (trans, C3), 27.4 (cis, C6), 27.3 (trans, C6), 19.73 (cis, C5), 19.71 (trans, C5)

MS (EI): 365, $363\left(\mathrm{M}^{+}-\mathrm{C}_{4} \mathrm{H}_{9}\right)$<smiles>FC(Br)=Cc1ccc(Br)cc1</smiles>

${ }^{1} \mathrm{H}$ NMR (300 MHz, $\left.\mathrm{CDCl}_{3}\right)$ :

7.5-7.4 (m, 2H, ArH), 7.4-7.2 (2d, J=8.5Hz, J'=8.7Hz, $2 \mathrm{H}, A r H), 6.5-6.6\left(\mathrm{~d}, 0.5 \mathrm{H},{ }^{3} \mathrm{~J}^{\mathrm{cis}}{ }_{\mathrm{H}-}\right.$ $\mathrm{F}=17.0 \mathrm{~Hz}, \mathrm{H} 2), 6.0-5.8\left(\mathrm{~d}, 0.5 \mathrm{H},{ }^{3} \mathrm{~J}_{\mathrm{H}-\mathrm{F}}^{\text {trans }}=32.0 \mathrm{~Hz}, \mathrm{H} 2\right)$

${ }^{19} \mathrm{~F}$ NMR $\left(282.5 \mathrm{MHz}, \mathrm{CDCl}_{3}\right)$ :

$-64\left(\mathrm{~d},{ }^{3} \mathrm{~J}_{\mathrm{H}-\mathrm{F}}^{\mathrm{cis}}=17.0 \mathrm{~Hz}\right),-67\left(\mathrm{~d},{ }^{3} \mathrm{~J}_{\mathrm{H}-\mathrm{F}}^{\text {trans }}=32.0 \mathrm{~Hz}\right)$

${ }^{13} \mathrm{C}$ NMR (75.5 MHz, $\left.\mathrm{CDCl}_{3}\right)$ :

$135.9\left(\mathrm{~d},{ }^{1} \mathrm{~J}_{\mathrm{C}-\mathrm{F}}^{\mathrm{cis}}=317 \mathrm{~Hz}, \mathrm{C}_{1}\right), 135.0\left(\mathrm{~d},{ }^{2} \mathrm{~J}^{\text {trans }}{ }_{\mathrm{C}-\mathrm{F}}=331 \mathrm{~Hz}, \mathrm{C}_{1}\right), 132.2\left(\mathrm{C}_{5}\right), 131.7\left(\mathrm{~d},{ }^{6} \mathrm{~J}^{\text {trans }}{ }_{\mathrm{C}-}\right.$ $\left.\mathrm{F}=7.47 \mathrm{~Hz}, \mathrm{C}_{6}\right), 130.8\left(\mathrm{~d},{ }^{6} \mathrm{~J}^{\mathrm{cis}}{ }_{\mathrm{C}-\mathrm{F}}=8.0 \mathrm{~Hz}, \mathrm{C}_{6}\right), 130.1\left(\mathrm{~d},{ }^{4} \mathrm{~J}_{\mathrm{C}-\mathrm{F}}^{\mathrm{cis}}=3.2 \mathrm{~Hz}, \mathrm{C}_{4}\right), 122\left(\mathrm{~m}, \mathrm{C}_{3}\right), 112(\mathrm{~d}$, $\left.{ }^{2} \mathrm{~J}_{\mathrm{C}-\mathrm{F}}^{\text {trans }}=6.3 \mathrm{~Hz}, \mathrm{C}_{2}\right), 111\left(\mathrm{~d},{ }^{2} \mathrm{~J}_{\mathrm{C}-\mathrm{F}}^{\mathrm{cis}}=24.7 \mathrm{~Hz}, \mathrm{C}_{2}\right)$.

MS (EI): $282\left(\mathrm{M}^{+}, 50 \%\right), 280\left(\mathrm{M}^{+}, 100 \%\right), 278\left(\mathrm{M}^{+}, 50 \%\right), 201\left(\mathrm{M}^{+}-\mathrm{Br}, 20 \%\right), 199\left(\mathrm{M}^{+}-\mathrm{Br}\right.$, $20 \%)$ 
<smiles>FC(Br)=Cc1ccc(F)cc1</smiles>

${ }^{1} \mathrm{H} \mathrm{NMR}\left(300 \mathrm{MHz}, \mathrm{CDCl}_{3}\right)$ :

7.6-7.3 (m, 2H), 7.2-6.9 (m, 2H), 6.6-6.5 (d, $\left.{ }^{2} \mathrm{~J}_{\mathrm{H}-\mathrm{F}}^{\mathrm{cis}}=14.6 \mathrm{~Hz}, 0.5 \mathrm{H}, \mathrm{H} 2\right), 6.1-5.8\left(\mathrm{~d},{ }^{2} \mathrm{~J}^{\text {trans }}{ }_{\mathrm{H}-}\right.$ $\mathrm{F}=32.5 \mathrm{~Hz}, 0.5 \mathrm{H}, \mathrm{H} 2)$

${ }^{19} \mathrm{~F}$ NMR $\left(282.5 \mathrm{MHz}, \mathrm{CDCl}_{3}\right)$ :

$-66.2 \mathrm{ppm}\left(\mathrm{d},{ }^{2} \mathrm{~J}_{\mathrm{H}-\mathrm{F}}^{\mathrm{cis}}=14.6 \mathrm{~Hz}, 0.5 \mathrm{~F}\right),-69.6 \mathrm{ppm}\left(\mathrm{d},{ }^{2} \mathrm{~J}_{\mathrm{H}-\mathrm{F}}^{\mathrm{trans}}=14.6 \mathrm{~Hz}, 0.5 \mathrm{~F}\right),-113.3 \mathrm{ppm}(\mathrm{m}, 1 \mathrm{~F})$.

${ }^{13} \mathrm{C}$ NMR (75.5 MHz, $\left.\mathrm{CDCl}_{3}\right)$ :

$162.7\left(\mathrm{~d},{ }^{1} \mathrm{~J}=248.4 \mathrm{~Hz}, \mathrm{C}_{6}{ }^{\text {cis }}\right), 162.4\left(\mathrm{~d},{ }^{1} \mathrm{~J}=248.4 \mathrm{~Hz}, \mathrm{C}_{6}{ }^{\text {trans }}\right), 135.1\left(\mathrm{~d},{ }^{1} \mathrm{~J}=317.4 \mathrm{~Hz}, \mathrm{C}_{1}{ }^{\text {cis }}\right), 134.0$ $\left(\mathrm{d},{ }^{1} \mathrm{~J}=330.0 \mathrm{~Hz}, \mathrm{C}_{1}{ }^{\text {trans }}\right), 130.5\left(\mathrm{dd}, \mathrm{J}=8.0 \mathrm{~Hz}, \mathrm{~J}=2.9 \mathrm{~Hz}, \mathrm{C}_{4}{ }^{\text {cis }}\right), 130.2\left(\mathrm{dd}, \mathrm{J}=7.8 \mathrm{~Hz}, \mathrm{~J}{ }^{\prime}=7.8 \mathrm{~Hz}\right.$, $\left.\mathrm{C}_{4}{ }^{\text {trans }}\right), 129.1\left(\mathrm{C}_{3}{ }^{\text {trans }}\right), 127.9\left(\mathrm{C}_{3}{ }^{\text {cis }}, 116.1\left(\mathrm{~d}, \mathrm{~J}=21.8 \mathrm{~Hz}, \mathrm{C}_{5}{ }^{\text {trans }}\right), 115.9\left(\mathrm{~d}, \mathrm{~J}=21.3 \mathrm{~Hz}, \mathrm{C}_{5}{ }^{\mathrm{cis}}\right)\right.$, $112.4\left(\mathrm{~d},{ }^{2} \mathrm{~J}=6.3 \mathrm{~Hz}, \mathrm{C}_{2}{ }^{\text {trans }}\right), 111.2\left(\mathrm{~d},{ }^{2} \mathrm{~J}=24.7 \mathrm{~Hz}, \mathrm{C}_{2}{ }^{\text {cis }}\right)$<smiles>O=[N+]([O-])c1ccc(C=C(F)F)cc1</smiles>

${ }^{1} \mathrm{H}$ NMR (300 MHz, $\left.\mathrm{CDCl}_{3}\right)$ :

$8.2\left(2 \mathrm{~d}, 2 \mathrm{H}, \mathrm{J}=8.7 \mathrm{~Hz}, \mathrm{~J}^{\prime}=8.7 \mathrm{~Hz}, \mathrm{H} 5\right), 7.6-7.4(2 \mathrm{~d}, \mathrm{~J}=8.7 \mathrm{~Hz}, \mathrm{~J}=8.7 \mathrm{~Hz}, 2 \mathrm{H}, \mathrm{H} 4), 6.7(\mathrm{~d}, 0.5 \mathrm{H}$, $\left.{ }^{3} \mathrm{~J}_{\mathrm{H}-\mathrm{F}}^{\mathrm{cis}}=14.4 \mathrm{~Hz}, \mathrm{H} 2\right), 6.0\left(\mathrm{~d}, 0.5 \mathrm{H},{ }^{3} \mathrm{~J}_{\mathrm{H}-\mathrm{F}}^{\text {trans }}=31.9 \mathrm{~Hz}, \mathrm{H} 2\right)$

${ }^{19} \mathrm{~F}$ NMR $\left(282.5 \mathrm{MHz}, \mathrm{CDCl}_{3}\right)$ :

$-59.3\left(\mathrm{~d},{ }^{3} \mathrm{~J}_{\mathrm{H}-\mathrm{F}}^{\mathrm{cis}}=14.4 \mathrm{~Hz}\right),-62.3\left(\mathrm{~d},{ }^{3} \mathrm{~J}_{\mathrm{H}-\mathrm{F}}^{\text {trans }}=31.9 \mathrm{~Hz}\right)$

${ }^{13} \mathrm{C} \mathrm{NMR}\left(75.5 \mathrm{MHz}, \mathrm{CDCl}_{3}\right)$ :

145.9 (cis, $\mathrm{C}_{6}$ ), 145.6 (trans, $\left.\mathrm{C}_{6}\right), 136.4\left(\mathrm{~d},{ }^{2} \mathrm{~J}^{\mathrm{ciss}}{ }_{\mathrm{C}-\mathrm{F}}=320 \mathrm{~Hz}, \mathrm{C}_{1}\right), 137.6\left(\mathrm{~d},{ }^{3}{ }^{\text {trans }}{ }_{\mathrm{C}-\mathrm{F}}=5.2 \mathrm{~Hz}, \mathrm{C}_{3}\right)$, $137.2\left(\mathrm{~d},{ }^{3} \mathrm{~J}_{\mathrm{C}-\mathrm{F}}^{\mathrm{cis}}=8.6 \mathrm{~Hz}, \mathrm{C}_{3}\right), 136.2\left(\mathrm{~d},{ }^{1} \mathrm{~J}^{\text {trans }}{ }_{\mathrm{C}-\mathrm{F}}=334 \mathrm{~Hz}, \mathrm{C}_{1}\right), 127.9\left(\mathrm{~d},{ }^{5} \mathrm{~J}_{\mathrm{C}-\mathrm{F}}^{\mathrm{cis}}=2.9 \mathrm{~Hz}, \mathrm{C}_{5}\right)$, $127.5\left(\mathrm{~d},{ }^{5}{ }^{\text {trans }}{ }_{\mathrm{C}-\mathrm{F}}=8.0 \mathrm{~Hz}, \mathrm{C}_{5}\right), 122.9\left(\mathrm{C}_{4}\right), 122.4\left(\mathrm{~d},{ }^{4} \mathrm{~J}_{\mathrm{C}-\mathrm{F}}^{\mathrm{cis}}=24.1 \mathrm{~Hz}, \mathrm{C}_{4}\right), 110.5\left(\mathrm{~d},{ }^{2} \mathrm{~J}^{\text {trans }}{ }_{\mathrm{C}-}\right.$ $\left.\mathrm{F}=5.7 \mathrm{~Hz}, \mathrm{C}_{2}\right), 109.4\left(\mathrm{~d},{ }^{2} \mathrm{~J}_{\mathrm{C}-\mathrm{F}}^{\mathrm{cis}}=26.4 \mathrm{~Hz}, \mathrm{C}_{2}\right)$

MS (EI): 347, $245\left(\mathrm{M}^{+}\right), 217,215\left(\mathrm{M}^{+}-\mathrm{NO}\right), 201,199\left(\mathrm{M}^{+}-\mathrm{NO}_{2}\right)$ 
<smiles>COc1ccc(C=C(F)F)cc1</smiles>

${ }^{1} \mathrm{H}$ NMR $\left(300 \mathrm{MHz}, \mathrm{CDCl}_{3}\right)$ :

7.4-7.2 (2d, 2H, J=8.7Hz, J'=8.4Hz, H4), 7.0-6.7 (2d, J=8.7Hz, J'=8.4Hz, 2H, H5), 6.5 (d, $\left.0.5 \mathrm{H},{ }^{3}{ }_{\mathrm{H}-\mathrm{F}}^{\mathrm{cis}}=15.0 \mathrm{~Hz}, \mathrm{H} 2\right), 5.8\left(\mathrm{~d}, 0.5 \mathrm{H},{ }^{3} \mathrm{~J}_{\mathrm{H}-\mathrm{F}}^{\text {trans }}=33.3 \mathrm{~Hz}, \mathrm{H} 2\right), 3.7(2 \mathrm{~s}, 3 \mathrm{H}, \mathrm{H} 7)$

${ }^{19} \mathrm{~F}$ NMR $\left(282.5 \mathrm{MHz}, \mathrm{CDCl}_{3}\right)$ :

$-68.2\left(\mathrm{~d},{ }^{3} \mathrm{~J}_{\mathrm{H}-\mathrm{F}}^{\mathrm{cis}}=15.0 \mathrm{~Hz}\right),-71.4\left(\mathrm{~d},{ }^{3} \mathrm{~J}_{\mathrm{H}-\mathrm{F}}^{\text {trans }}=33.3 \mathrm{~Hz}\right)$

${ }^{13} \mathrm{C}$ NMR (75.5 MHz, $\left.\mathrm{CDCl}_{3}\right)$ :

$159.0\left(\mathrm{~d},{ }^{6} \mathrm{~J}^{\text {trans }}{ }_{\mathrm{C}-\mathrm{F}}=2.9 \mathrm{~Hz}, \mathrm{C}_{6}\right), 136.0-130.5\left(2 \mathrm{~d},{ }^{1} \mathrm{~J}_{\mathrm{C}-\mathrm{F}}^{\mathrm{cis}}=314 \mathrm{~Hz},{ }^{1}{ }^{\text {trans }}{ }_{\mathrm{C}-\mathrm{F}}=329 \mathrm{~Hz}, \mathrm{C}_{1}\right), 129.0(\mathrm{~d}$, ${ }^{4} \mathrm{~J}_{\text {trans }}$ C- $\left.\left.=7.1 \mathrm{~Hz}, \mathrm{C}_{4}\right), 125.0-123.0\left(2 \mathrm{~d},{ }^{3} \mathrm{~J}_{\mathrm{C}-\mathrm{F}}^{\mathrm{cis}}=8.0 \mathrm{~Hz},{ }^{3} \mathrm{~J}^{\text {trans }}{ }_{\mathrm{C}-\mathrm{F}}=4.0 \mathrm{~Hz} \mathrm{C}\right)_{3}\right), 114.3-114.5(\mathrm{~d}$, $\left.{ }^{5} \mathrm{~J}_{\mathrm{C}-\mathrm{F}}^{\text {trans }}=4.5 \mathrm{~Hz}, \mathrm{C}_{5}\right), 113.1\left(\mathrm{~d},{ }^{2} \mathrm{~J}_{\mathrm{C}-\mathrm{F}}^{\text {trans }}=6.9 \mathrm{~Hz}, \mathrm{C}_{2}\right), 111.2\left(\mathrm{~d},{ }^{2}{ }^{\mathrm{cis}}{ }_{\mathrm{C}-\mathrm{F}}=24 \mathrm{~Hz}, \mathrm{C}_{2}\right), 56\left(\mathrm{C}_{7}\right)$

MS (EI): 232, $230\left(\mathrm{M}^{+}\right), 217,215\left(\mathrm{M}^{+}-\mathrm{CH}_{3}\right)$<smiles>O=[N+]([O-])c1ccccc1/C=C(/F)C(F)Br</smiles>

${ }^{1} \mathrm{H}$ NMR $\left(300 \mathrm{MHz}, \mathrm{CDCl}_{3}\right)$ :

8.1-7.9 (m, 1H, ArH), 7.7-7.3 (m, 3H, ArH), 7.1-6.9 (d, 0.7H, $\left.{ }^{3} \mathrm{~J}_{\mathrm{H}-\mathrm{F}}^{\mathrm{cis}}=11.8 \mathrm{~Hz}\right), 6.6-6.4(\mathrm{~d}$, $\left.0.3 \mathrm{H},{ }^{3} \mathrm{~J}_{\mathrm{H}-\mathrm{F}}^{\text {trans }}=30.1 \mathrm{~Hz}\right)$

${ }^{19} \mathrm{~F}$ NMR $\left(282.5 \mathrm{MHz}, \mathrm{CDCl}_{3}\right):-64.3\left(\mathrm{~d},{ }^{3} \mathrm{~J}_{\mathrm{H}-\mathrm{F}}^{\mathrm{cis}}=11.8 \mathrm{~Hz}\right),-67.6\left(\mathrm{~d},{ }^{3} \mathrm{~J}_{\mathrm{H}-\mathrm{F}}^{\mathrm{trans}}=30.1 \mathrm{~Hz}\right)$

${ }^{13} \mathrm{C}$ NMR (75.5 MHz, $\left.\mathrm{CDCl}_{3}\right)$ :

147.3 (cis, $\left.\mathrm{C}_{4}\right), 146.6$ (trans, $\left.\mathrm{C}_{4}\right), 137.0\left({ }^{1} \mathrm{~J}_{\mathrm{C}-\mathrm{F}}^{\mathrm{cis}}=319 \mathrm{~Hz}, \mathrm{C}_{1}\right), 133.7$ (trans, $\left.\mathrm{C}_{8}\right), 135.5(\mathrm{~d}$, $\left.{ }^{1} \mathrm{~J}_{\mathrm{CF}}^{\text {trans }}=332 \mathrm{~Hz}, \mathrm{C}_{1}\right), 133.8\left(\mathrm{cis}, \mathrm{C}_{8}\right), 132.4\left(\mathrm{~d}, \mathrm{~J}_{\mathrm{C}-\mathrm{F}}^{\mathrm{cis}}=1.8 \mathrm{~Hz}, \mathrm{C}_{6}\right), 131.5\left(\mathrm{~d}, \mathrm{~J}^{\text {trans }} \mathrm{C}-\mathrm{F}=9.2 \mathrm{~Hz}, \mathrm{C}_{6}\right)$, $128.8\left(\mathrm{C}_{7}, \mathrm{cis}\right), 128.3\left(\mathrm{C}_{7}\right.$, trans), $127.1\left(\mathrm{~d},{ }^{3} \mathrm{~J}_{\mathrm{C}-\mathrm{F}}^{\mathrm{cis}}=9.2 \mathrm{~Hz}, \mathrm{C}_{3}\right), 126.0\left(\mathrm{~d},{ }^{3} \mathrm{~J}^{\text {trans }}{ }_{\mathrm{C}-\mathrm{F}}=4.1 \mathrm{~Hz}, \mathrm{C}_{3}\right)$, $124.0\left(\mathrm{C}_{5}\right), 107.7\left(\mathrm{~d},{ }^{2} \mathrm{~J}_{\mathrm{C}-\mathrm{F}}^{\mathrm{trans}}=5.1 \mathrm{~Hz}, \mathrm{C}_{2}\right), 109.0\left(\mathrm{~d},{ }^{2} \mathrm{~J}_{\mathrm{C}-\mathrm{F}}^{\mathrm{cis}}=28.2 \mathrm{~Hz}, \mathrm{C}_{2}\right)$,

MS (EI): 247, $245\left(\mathrm{M}^{+}\right), 166\left(\mathrm{M}^{+}-\mathrm{Br}\right)$ 
<smiles>COc1ccccc1C=C(C)C(=O)O</smiles>

${ }^{1} \mathrm{H}$ NMR $\left(300 \mathrm{MHz}, \mathrm{CDCl}_{3}\right)$ :

7.6-7.5 (d, J=8.0Hz, 1H, ArH), 7.3-7.1 (m, 1H, ArH), 6.9-6.8 (m, 1H, ArH), $6.8(\mathrm{~d}, \mathrm{~J}=8.1 \mathrm{~Hz}$, $1 \mathrm{H}, \mathrm{ArH}), 6.7\left(\mathrm{~d},{ }^{3} \mathrm{~J}_{\mathrm{H}-\mathrm{F}}^{\mathrm{cis}}=15.1 \mathrm{~Hz}, 0.5 \mathrm{H}, \mathrm{H} 2\right), 6.3\left(\mathrm{~d}, 0.5 \mathrm{H},{ }^{3} \mathrm{~J}_{\mathrm{H}-\mathrm{F}}^{\text {trans }}=34.3 \mathrm{~Hz}, \mathrm{H} 2\right), 3.7(\mathrm{~s}, 3 \mathrm{H}$, H9)

${ }^{19} \mathrm{~F}$ NMR $\left(282.5 \mathrm{MHz}, \mathrm{CDCl}_{3}\right)$ :

$-65.6\left(\mathrm{~d},{ }^{3} \mathrm{~J}_{\mathrm{H}-\mathrm{F}}^{\mathrm{cis}}=15.1 \mathrm{~Hz}\right),-69.5\left(\mathrm{~d},{ }^{3} \mathrm{~J}_{\mathrm{H}-\mathrm{F}}^{\mathrm{trans}}=34.3 \mathrm{~Hz}\right)$

${ }^{13} \mathrm{C}$ NMR (75.5 MHz, $\left.\mathrm{CDCl}_{3}\right)$ :

$157.2\left(\mathrm{~d}, \mathrm{~J}=3.5 \mathrm{~Hz}\right.$, cis, $\left.\mathrm{C}_{4}\right), 156.1$ (trans, $\left.\mathrm{C}_{4}\right), 135.5\left({ }^{1} \mathrm{~J}^{\mathrm{cis}}{ }_{\mathrm{C}-\mathrm{F}}=315 \mathrm{~Hz}, \mathrm{C}_{1}\right), 133.7(\mathrm{~d}$, $\left.{ }^{1} \mathrm{~J}^{\text {trans }}{ }_{\mathrm{CF}}=331 \mathrm{~Hz}, \mathrm{C}_{1}\right), 129.8$ (cis, ArH), 129.7 (trans, ArH), 129.5 (d, J=2.2Hz, cis, ArH), 129.3 $\left(\mathrm{d}, \mathrm{J}=1.8 \mathrm{~Hz}\right.$, trans, ArH), $121.7\left(\mathrm{~d},{ }^{3} \mathrm{~J}^{\text {trans }}{ }_{\mathrm{C}-\mathrm{F}}=5.2 \mathrm{~Hz}, \mathrm{C}_{3}\right), 121.1(\operatorname{trans}, \operatorname{ArH}), 120.8\left(\mathrm{~d},{ }^{3} \mathrm{~J}^{\mathrm{cis}}{ }_{\mathrm{C}-}\right.$ $\left.\mathrm{F}=8.0 \mathrm{~Hz}, \mathrm{C}_{3}\right), 120.7(\mathrm{cis}, \mathrm{ArH}), 111.0(\operatorname{trans} \mathrm{ArH}), 110.8(\mathrm{cis}, \mathrm{ArH}), 107.7\left(\mathrm{~d},{ }^{2} \mathrm{~J}^{\mathrm{cis}}{ }_{\mathrm{C}-\mathrm{F}}=24.7 \mathrm{~Hz}\right.$, $\left.\mathrm{C}_{2}\right), 107.3\left(\mathrm{~d},{ }^{2} \mathrm{~J}_{\mathrm{C}-\mathrm{F}}^{\text {trans }}=4.6 \mathrm{~Hz}, \mathrm{C}_{2}\right), 55.9-55.8(\mathrm{C} 9$, cis, trans)

MS (EI): 232, $230\left(\mathrm{M}^{+}\right), 151\left(\mathrm{M}^{+}-\mathrm{Br}\right)$<smiles>FC(=[V])c1ccc2c(c1)OCO2</smiles>

${ }^{1} \mathrm{H}$ NMR $\left(300 \mathrm{MHz}, \mathrm{CDCl}_{3}\right)$ :

$7.1(\mathrm{~d}, 0.5 \mathrm{H}, \mathrm{J}=1.5 \mathrm{~Hz}, \mathrm{H} 4 \mathrm{cis}), 7.0(\mathrm{~d}, 0.5 \mathrm{H}, \mathrm{J}=1.0 \mathrm{~Hz}, \mathrm{H} 4$ trans), 6.9-6.7 (m, 2H, H7, H8), 6.6 $\left(\mathrm{d},{ }^{3} \mathrm{~J}_{\mathrm{H}-\mathrm{F}}^{\mathrm{cis}}=15.4 \mathrm{~Hz}, 0.5 \mathrm{H}, \mathrm{H} 2\right), 5.97-5.95\left(2 \mathrm{~s}, 2 \mathrm{H}\right.$, trans, cis, H9), $5.9\left(\mathrm{~d},{ }^{3} \mathrm{~J}_{\mathrm{H}-\mathrm{F}}^{\text {trans }}=32.5 \mathrm{~Hz}\right.$, $0.5 \mathrm{H}, \mathrm{H} 2)$

${ }^{19} \mathrm{~F}$ NMR $\left(282.5 \mathrm{MHz}, \mathrm{CDCl}_{3}\right)$ :

$-67.6\left(\mathrm{~d},{ }^{3} \mathrm{~J}_{\mathrm{H}-\mathrm{F}}^{\mathrm{cis}}=15.4 \mathrm{~Hz}\right),-70.6\left(\mathrm{~d},{ }^{3} \mathrm{~J}_{\mathrm{H}-\mathrm{F}}^{\mathrm{trans}}=32.5 \mathrm{~Hz}\right)$

${ }^{13} \mathrm{C} \mathrm{NMR}\left(75.5 \mathrm{MHz}, \mathrm{CDCl}_{3}\right)$ :

148.3 (C5, trans), 148.1 (C5, cis), 147.8 (C6, cis), 147.6 (d, J=2.9Hz, C6, trans), 136.5-132.3 $\left(\mathrm{d},{ }^{1} \mathrm{~J}^{\mathrm{cis}}=314 \mathrm{~Hz}, \mathrm{C} 1\right), 135.1-130.8\left(\mathrm{~d},{ }^{1} \mathrm{~J}^{\text {trans }}=329 \mathrm{~Hz}, \mathrm{C} 1\right), 127.01-127.10\left(\mathrm{~d},{ }^{3} \mathrm{~J}^{\text {trans }}=4.6 \mathrm{~Hz}, \mathrm{C} 3\right)$, $125.5-125.4\left(\mathrm{~d},{ }^{3} \mathrm{~J}^{\mathrm{cis}}=8.6 \mathrm{~Hz}, \mathrm{C} 3\right), 123.3\left(\mathrm{~d},{ }^{4} \mathrm{~J}^{\mathrm{cis}}=3.7 \mathrm{~Hz}, \mathrm{C} 8\right), 122.8\left(\mathrm{~d},{ }^{4} \mathrm{~J}^{\text {trans }}=5.9 \mathrm{~Hz}, \mathrm{C} 8\right), 108.8$ (C7), $113.1\left(\mathrm{~d},{ }^{2} \mathrm{~J}^{\text {trans }}{ }_{\mathrm{C}-\mathrm{F}}=5.8 \mathrm{~Hz}, \mathrm{C} 2\right), 111.9-111.6\left(\mathrm{~d},{ }^{2} \mathrm{~J}_{\mathrm{C}-\mathrm{F}}^{\mathrm{cis}}=24.1 \mathrm{~Hz}, \mathrm{C} 2\right), 108.6-108.4(2 \mathrm{~d}$, C4, cis, trans), 101 (C9)

MS (EI): 246, $244\left(\mathrm{M}^{+}\right), 165\left(\mathrm{M}^{+}-\mathrm{Br}\right)$ 
<smiles>COC(=O)c1ccc(C=C(F)F)cc1</smiles>

${ }^{1} \mathrm{H}$ NMR $\left(300 \mathrm{MHz}, \mathrm{CDCl}_{3}\right)$ :

8.1-7.9 (b, 2H, H5cis, trans), 7.6 (d, J=8.4Hz, H4cis), 7.5-7.4 (d, J=8.5Hz, H4trans), 6.7 (d, $\left.{ }^{3} \mathrm{~J}_{\mathrm{H}-\mathrm{F}}^{\mathrm{cis}}=14.9 \mathrm{~Hz}, 0.5 \mathrm{H}, \mathrm{H} 2\right), 6.0\left(\mathrm{~d},{ }^{3} \mathrm{~J}_{\mathrm{H}-\mathrm{F}}^{\mathrm{trans}}=32.3 \mathrm{~Hz}, 0.5 \mathrm{H}, \mathrm{H} 2\right), 4.0-3.8(2 \mathrm{~s}, 3 \mathrm{H}, \mathrm{H} 8 \mathrm{cis}$, trans $)$

${ }^{19} \mathrm{~F}$ NMR $\left(282.5 \mathrm{MHz}, \mathrm{CDCl}_{3}\right)$ :

$-62.2\left(\mathrm{~d},{ }^{3} \mathrm{~J}_{\mathrm{H}-\mathrm{F}}^{\mathrm{cis}}=14.9 \mathrm{~Hz}\right),-64.5\left(\mathrm{~d},{ }^{3} \mathrm{~J}_{\mathrm{H}-\mathrm{F}}^{\mathrm{trans}}=32.3 \mathrm{~Hz}\right)$

${ }^{13} \mathrm{C}$ NMR (75.5 MHz, $\left.\mathrm{CDCl}_{3}\right)$ :

$167.91\left(\mathrm{C}_{7}\right.$, cis $), 167.87\left(\mathrm{C}_{7}\right.$, trans $), 138.1\left(\mathrm{~d},{ }^{6} \mathrm{~J}^{\text {trans }}{ }_{\mathrm{C}-\mathrm{F}}=5.2 \mathrm{~Hz}, \mathrm{C}_{6}\right), 137.6\left(\mathrm{~d},{ }^{2} \mathrm{~J}_{\mathrm{C}-\mathrm{F}}^{\mathrm{cis}}=318 \mathrm{~Hz}\right.$, $\left.\mathrm{C}_{1}\right), 137.4\left(\mathrm{~d},{ }^{6} \mathrm{~J}_{\mathrm{C}-\mathrm{F}}^{\mathrm{cis}}=8.6 \mathrm{~Hz}, \mathrm{C}_{6}\right), 137.1\left(\mathrm{~d},{ }^{1} \mathrm{~J}^{\text {trans }}{ }_{\mathrm{C}-\mathrm{F}}=333.5 \mathrm{~Hz}, \mathrm{C}_{1}\right), 131.3\left(\mathrm{C}_{5}\right.$, trans $), 131.0\left(\mathrm{C}_{5}\right.$, cis), $130.7\left(\mathrm{C}_{3}\right.$, cis $), 130.5\left(\mathrm{~d}, \mathrm{~J}^{\mathrm{trans}}{ }_{\mathrm{C}-\mathrm{F}}=2.9 \mathrm{~Hz}, \mathrm{C}_{3}\right), 129.6\left(\mathrm{~d},{ }^{4} \mathrm{~J}_{\mathrm{C}-\mathrm{F}}=2.9 \mathrm{~Hz}, \mathrm{C}_{4}\right), 129.2(\mathrm{~d}$, $\left.{ }_{\mathrm{J}}^{4 \text { trans }}{ }_{\mathrm{C}-\mathrm{F}}=7.5 \mathrm{~Hz}, \mathrm{C}_{4}\right), 113.8\left(\mathrm{~d}, \mathrm{~J}^{2}{ }_{\mathrm{C}-\mathrm{F}}^{\mathrm{r}}=5.6 \mathrm{~Hz}, \mathrm{C}_{2}\right), 112.5\left(\mathrm{~d},{ }^{2} \mathrm{~J}_{\mathrm{C}-\mathrm{F}}^{\mathrm{cis}}=24.7 \mathrm{~Hz}, \mathrm{C}_{2}\right), 53.5\left(\mathrm{C}_{8}\right)$<smiles>FC(F)=Cc1ccc2ccccc2c1</smiles>

${ }^{1} \mathrm{H}$ NMR $\left(300 \mathrm{MHz}, \mathrm{CDCl}_{3}\right)$ :

8.0-7.7 (m, 4H), 707-7.3 (m, 3H), 6.9-6.7 $\left(\mathrm{d},{ }^{3} \mathrm{~J}_{\mathrm{H}-\mathrm{F}}^{\mathrm{cis}}=15.1 \mathrm{~Hz}, 0.5 \mathrm{H}, \mathrm{H} 2\right), 6.2-6.0\left(\mathrm{~d},{ }^{3}{ }^{\mathrm{trans}}{ }_{\mathrm{H}-}\right.$ $\mathrm{F}=32.8 \mathrm{~Hz}, 0.5 \mathrm{H}, \mathrm{H} 2$ )

${ }^{19} \mathrm{~F}$ NMR $\left(282.5 \mathrm{MHz}, \mathrm{CDCl}_{3}\right)$ :

$-65.1\left(\mathrm{~d},{ }^{3} \mathrm{~J}_{\mathrm{H}-\mathrm{F}}^{\mathrm{cis}}=15.1 \mathrm{~Hz}\right),-67.9\left(\mathrm{~d},{ }^{3} \mathrm{~J}_{\mathrm{H}-\mathrm{F}}^{\text {trans }}=32.8 \mathrm{~Hz}\right)$, cis/trans $=1 / 0.93$

${ }^{13} \mathrm{C}$ NMR (75.5 MHz, $\mathrm{CDCl}_{3}$ ):

$135.6\left(\mathrm{~d},{ }^{1} \mathrm{~J}^{\mathrm{cis}}{ }_{\mathrm{C}-\mathrm{F}}=316 \mathrm{~Hz}, \mathrm{C}_{1}\right), 134.5\left(\mathrm{~d},{ }^{1} \mathrm{~J}^{\text {trans }}{ }_{\mathrm{C}-\mathrm{F}}=331.8 \mathrm{~Hz}, \mathrm{C}_{1}\right), 133.7\left(\mathrm{C}_{11}\right.$, trans $), 133.6\left(\mathrm{C}_{11}\right.$, cis $), 133.2\left(\mathrm{C}_{3}, \mathrm{cis}\right), 133.1\left(\mathrm{~d}, \mathrm{C}_{3}, \mathrm{~J}^{\text {trans }}=1.7 \mathrm{~Hz}\right), 130.4\left(\mathrm{~d}, \mathrm{C}_{6}, \mathrm{~J}^{\text {trans }}=4.6 \mathrm{~Hz}\right), 129.3\left(\mathrm{~d}, \mathrm{C}_{6}\right.$, $\left.\mathrm{J}^{\mathrm{cis}}=8.6 \mathrm{~Hz}\right), 128.8\left(\mathrm{C}_{\mathrm{Ar}} \mathrm{H}\right.$, trans $), 128.6\left(\mathrm{C}_{\mathrm{Ar}} \mathrm{H}\right.$, trans $), 128.5\left(\mathrm{C}_{\mathrm{Ar}} \mathrm{H}\right.$, cis $), 128.4\left(\mathrm{C}_{\mathrm{Ar}} \mathrm{H}\right.$, cis $)$, $128.3\left(\mathrm{~d}, \mathrm{C}_{12}, \mathrm{~J}^{\text {cis }}=4.1 \mathrm{~Hz}\right), 128.1\left(\mathrm{C}_{\mathrm{Ar}} \mathrm{H}, \mathrm{cis}\right), 128.0\left(\mathrm{C}_{\mathrm{Ar}} \mathrm{H}\right.$, trans $), 127.8\left(\mathrm{~d}, \mathrm{C}_{12}, \mathrm{~J}^{\text {trans }}=6.9 \mathrm{~Hz}\right)$, $126.9\left(\mathrm{C}_{8}, \mathrm{C}_{9}\right.$, cis, trans), $126.3\left(\mathrm{~d}, \mathrm{C}_{4}, \mathrm{~J}^{\mathrm{cis}}=2.7 \mathrm{~Hz}\right), 126.0\left(\mathrm{~d}, \mathrm{C}_{4}, \mathrm{~J}^{\mathrm{trans}}=7.5 \mathrm{~Hz}\right), 113.7\left(\mathrm{~d}, \mathrm{C}_{2}\right.$, $\left.\mathrm{J}^{\text {trans }}=6.0 \mathrm{~Hz}\right), 112.3\left(\mathrm{~d}, \mathrm{C}_{2}, \mathrm{~J}^{\mathrm{cis}}=24.2 \mathrm{~Hz}\right)$ 
<smiles>FC(Br)=C(c1ccccc1)c1ccccc1</smiles>

${ }^{1} \mathrm{H}$ NMR $\left(300 \mathrm{MHz}, \mathrm{CDCl}_{3}\right)$ :

7.2-7.5 (m, 10H, Ar-H)

${ }^{19} \mathrm{~F} \mathrm{NMR}\left(282.5 \mathrm{MHz}, \mathrm{CDCl}_{3}\right)$ :

$-71.5 \mathrm{ppm}$

${ }^{13} \mathrm{C} \mathrm{NMR}\left(75.5 \mathrm{MHz}, \mathrm{CDCl}_{3}\right)$ :

138.7 (d, J=4.0Hz, $\left.\mathrm{C}_{14}\right), 136.6\left(\mathrm{~d}, \mathrm{~J}=2.3 \mathrm{~Hz}, \mathrm{C}_{3}\right), 134.3-132.1\left(\mathrm{~d}, \mathrm{~J}=325 \mathrm{~Hz}, \mathrm{C}_{1}\right), 130.5$ (d, $\mathrm{J}=3.7 \mathrm{~Hz}), 129.6(\mathrm{~d}, \mathrm{~J}=4.6 \mathrm{~Hz}), 128.9,128.7,128.4,128.2,125.2-125.0\left(\mathrm{~d}, \mathrm{~J}=10.9 \mathrm{~Hz}, \mathrm{C}_{2}\right)$

MS (EI): 278, $276\left(\mathrm{M}^{+}\right), 197(\mathrm{M}-\mathrm{Br}), 196\left(\mathrm{M}^{+}-\mathrm{HBr}\right)$.

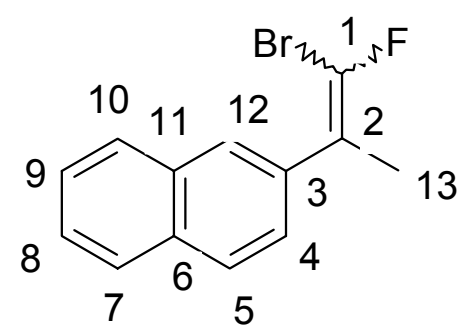

${ }^{1} \mathrm{H}$ NMR (300 MHz, $\left.\mathrm{CDCl}_{3}\right)$ :

7.9-7.6 (m, 4H), 7.5-7.3 (m, 3H), 2.2-2.0 (m, 3H, H13)

${ }^{19} \mathrm{~F}$ NMR (282.5 MHz.CDCl 3$)$ :

$-74.4\left(\mathrm{~m}, \mathrm{~A}\right.$ and $\mathrm{B}$ cannot be resoluted by ${ }^{19} \mathrm{~F}$ NMR)

${ }^{13} \mathrm{C}$ NMR (75.5MHz, $\left.\mathrm{CDCl}_{3}\right)$ :

$136.7\left(\mathrm{C}_{\mathrm{Ar}}{ }^{\mathrm{q}}, \mathrm{C}_{3}, \mathrm{~B}\right), 133.6\left(\mathrm{C}_{\mathrm{Ar}}{ }^{\mathrm{q}}, \mathrm{C}_{3}, \mathrm{~B}\right), 134.3\left(\mathrm{C}_{\mathrm{Ar}}{ }^{\mathrm{q}}, \mathrm{A}\right), 133.6\left(\mathrm{C}_{\mathrm{Ar}}{ }^{\mathrm{q}}, \mathrm{B}\right), 133.1\left(\mathrm{C}_{\mathrm{Ar}}{ }^{\mathrm{q}}, \mathrm{A}\right)$, 133.0 ( $\left.\mathrm{C}_{\mathrm{Ar}}^{\mathrm{q}}, \mathrm{A}\right), 133.0\left(\mathrm{~d}, \mathrm{~J}=316.8 \mathrm{~Hz}, \mathrm{C}_{1}, \mathrm{~A}\right), 131.4\left(\mathrm{~d}, \mathrm{~J}=322 \mathrm{~Hz}, \mathrm{C}_{1}, \mathrm{~B}\right), 128.6\left(\mathrm{C}_{\mathrm{Ar}} \mathrm{H}, \mathrm{A}, \mathrm{B}\right)$, $128.5\left(\mathrm{C}_{\mathrm{Ar}} \mathrm{H}, \mathrm{A}\right), 128.4\left(\mathrm{C}_{\mathrm{Ar}} \mathrm{H}, \mathrm{B}\right), 128.2\left(\mathrm{C}_{\mathrm{Ar}} \mathrm{H}, \mathrm{A}\right), 128.1\left(\mathrm{C}_{\mathrm{Ar}} \mathrm{H}, \mathrm{B}\right), 128.0(\mathrm{~d}, \mathrm{~J}=3.2 \mathrm{~Hz}$, $\left.\mathrm{C}_{\mathrm{Ar}} \mathrm{H}, \mathrm{A}\right), 127.4(\mathrm{~d}, \mathrm{~J}=4.1 \mathrm{~Hz}, \mathrm{CArH}, \mathrm{B}), 126.8\left(\mathrm{C}_{\mathrm{Ar}} \mathrm{H}, \mathrm{C}_{9}, \mathrm{C}_{10}, \mathrm{~A}, \mathrm{~B}\right), 126.6(\mathrm{~d}, \mathrm{~J}=3.2 \mathrm{~Hz}$, $\left.\mathrm{C}_{\mathrm{Ar}} \mathrm{H}, \mathrm{A}\right), 126.2\left(\mathrm{~d}, \mathrm{~J}=4.6 \mathrm{~Hz}, \mathrm{C}_{\mathrm{Ar}} \mathrm{H}, \mathrm{B}\right), 120.0\left(\mathrm{C}_{2}, \mathrm{~A}\right), 118.2\left(\mathrm{C}_{2}, \mathrm{~B}\right), 21.1\left(\mathrm{C}_{13}, \mathrm{~B}\right), 18.3\left(\mathrm{C}_{13}\right.$, A)

MS (EI): 266, $264\left(\mathrm{M}^{+}\right), 183\left(\mathrm{M}^{+}-\mathrm{Br}\right)$

IR (KBr): 3056, 2622, 1651, 1589, 1505, 1121, 1013, 857, 817, 747 


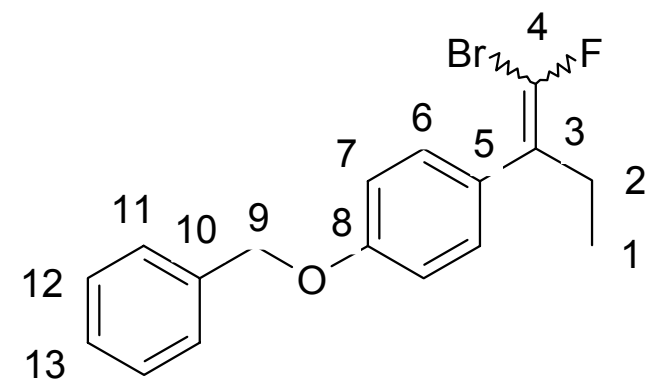

${ }^{1} \mathrm{H}$ NMR (300 MHz, $\left.\mathrm{CDCl}_{3}\right)$ :

7.4-7.2 (m, 5H), 7.2-7.0 (m, 2H), 7.0-6.8 (m, 2H), 5.0 (s, 2H, H9), 2.5-2.3 (m, 2H, H2), 1.0$0.8(\mathrm{q}, \mathrm{J}=7.8 \mathrm{~Hz}, 3 \mathrm{H}, \mathrm{H} 1)$

${ }^{19} \mathrm{~F}$ NMR (282.5 $\left.\mathrm{MHz} . \mathrm{CDCl}_{3}\right)$ :

-76.5 (A), $-76.7\left(\mathrm{t},{ }^{4} \mathrm{~J}_{\mathrm{H}-\mathrm{F}}=3.6 \mathrm{~Hz}, \mathrm{~B}\right), \mathrm{A} / \mathrm{B}=1 / 0.86$

${ }^{13} \mathrm{C} \mathrm{NMR}\left(75.5 \mathrm{MHz}, \mathrm{CDCl}_{3}\right)$ :

$158.7\left(\mathrm{C}_{8}, \mathrm{~A}, \mathrm{~B}\right), 137.2\left(\mathrm{C}_{10}, \mathrm{~A}, \mathrm{~B}\right), 131.4$ (d, J=315.7Hz, C 4 , A), 130.4 (d, J=2.9Hz, C, $\left.\mathrm{B}\right)$, 129.8 (d, J=3.4Hz, C,$A$ ), 129.4 (d, J=194.9Hz, C 4 , B), $129.0\left(\mathrm{C}_{11}, \mathrm{~A}, \mathrm{~B}\right), 128.7\left(\mathrm{C}_{5}, \mathrm{~A}, \mathrm{~B}\right)$, $128.4\left(\mathrm{C}_{13}, \mathrm{~A}, \mathrm{~B}\right), 128.0\left(\mathrm{C}_{12}, \mathrm{~B}\right), 127.9\left(\mathrm{C}_{12}, \mathrm{~A}\right), 125.6\left(\mathrm{C}_{3}, \mathrm{~B}\right), 123.5\left(\mathrm{~d}, \mathrm{~J}=6.3 \mathrm{~Hz}, \mathrm{C}_{3}, \mathrm{~A}\right)$, 115.0-114.9 ( $\left.\mathrm{C}_{6}, \mathrm{~A}, \mathrm{~B}\right), 70.9\left(\mathrm{C}_{9}\right), 28.2\left(\mathrm{C}_{2}, \mathrm{~B}\right), 25.5\left(\mathrm{~d}, \mathrm{~J}=3.7 \mathrm{~Hz}, \mathrm{C}_{2}, \mathrm{~A}\right), 12.9(\mathrm{~d}, \mathrm{~J}=2.3 \mathrm{~Hz}$, $\left.\mathrm{C}_{1}, \mathrm{~A}\right), 12.5-12.5\left(\mathrm{~d}, \mathrm{~J}=3.7 \mathrm{~Hz}, \mathrm{C}_{1}, \mathrm{~B}\right)$

MS (EI): 336, $334\left(\mathrm{M}^{+}\right), 240\left(\mathrm{M}^{+}-\mathrm{Br}\right)$

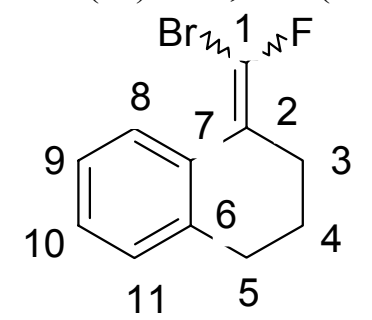

${ }^{1} \mathrm{H}$ NMR (300 MHz, $\left.\mathrm{CDCl}_{3}\right)$ :

7.9-7.7 (m, 1H), 7.4-7.1 (m, 3H), 2.9-2.7 (m, 3H, 1H3 + 2H5), 2.6-2.5 (m, 1H, 1H3), 2.0-1.8 (m, 2H, H4)

${ }^{19} \mathrm{~F}$ NMR (282.5 MHz.CDCl 3$)$ :

$-69\left(\mathrm{t}, 4 \mathrm{~J}_{\mathrm{H}-\mathrm{F}}=3.6 \mathrm{~Hz}\right),-72.6(\mathrm{~S}), \mathrm{A} / \mathrm{B}=1 / 1.6$

$\left.{ }^{13} \mathrm{C} \mathrm{NMR} \mathrm{(75.5MHz,} \mathrm{CDCl}_{3}\right)$ :

$22.7\left(\mathrm{~d},{ }^{4} \mathrm{~J}_{\mathrm{C}-\mathrm{F}}=2.3 \mathrm{~Hz}, \mathrm{~A}, \mathrm{C}_{4}\right), 23.2\left(\mathrm{~d},{ }^{4} \mathrm{~J}_{\mathrm{C}-\mathrm{F}}=1.4 \mathrm{~Hz}, \mathrm{~B}, \mathrm{C}_{4}\right), 26.0\left(\mathrm{~d},{ }^{5} \mathrm{~J}_{\mathrm{C}-\mathrm{F}}=6.4 \mathrm{HZ}, \mathrm{A}, \mathrm{C}_{3}\right), 30.65$ $\left(\mathrm{A}, \mathrm{C}_{5}\right), 30.6\left(\mathrm{~B}, \mathrm{C}_{3}\right), 31.1\left(\mathrm{~B}, \mathrm{C}_{5}\right), 116\left(\mathrm{~B}, \mathrm{C}_{2}\right), 119\left(\mathrm{~d},{ }^{2} \mathrm{~J}=17.0 \mathrm{~Hz}, \mathrm{~A}, \mathrm{C}_{2}\right), 125.8\left(\mathrm{~A}, \mathrm{C}_{\mathrm{Ar}}-\mathrm{H}\right)$, $126.6\left(\mathrm{~B}, \mathrm{C}_{\mathrm{Ar}}-\mathrm{H}\right), 127.9\left(\mathrm{~d}, \mathrm{~J}=1.4 \mathrm{~Hz}, \mathrm{~B}, \mathrm{C}_{\mathrm{Ar}}-\mathrm{H}\right), 128.1\left(\mathrm{~A}, \mathrm{C}_{\mathrm{Ar}} \mathrm{H}\right), 128.5\left(\mathrm{~B}, \mathrm{C}_{\mathrm{Ar}} \mathrm{H}\right), 129.0(\mathrm{~A}$, $\left.\left.\mathrm{C}_{\mathrm{Ar}} \mathrm{H}\right), 129.1\left(\mathrm{~B}, \mathrm{C}_{\mathrm{Ar}} \mathrm{H}\right), 129.2\left(\mathrm{~A}, \mathrm{C}_{\mathrm{Ar}} \mathrm{H}\right), 129.7\left(\mathrm{~d}, \mathrm{~J}=326.0 \mathrm{~Hz}, \mathrm{~A}, \mathrm{C}_{1}\right), \mathrm{B}, \mathrm{C}_{1}\right), 131.6(\mathrm{~d}$, $\left.\mathrm{J}=10.3 \mathrm{~Hz}, \mathrm{~A}, \mathrm{C}_{7}\right), 131.4-131.3\left(\mathrm{~d}, \mathrm{~J}=6.9 \mathrm{~Hz}, \mathrm{~B}, \mathrm{C}_{7}\right), 133.4\left(\mathrm{~d}, \mathrm{~J}=335.2 \mathrm{~Hz}, 135.9\left(\mathrm{C}_{6}, \mathrm{~B}\right) 140.3\right.$ $\left(\mathrm{d}, \mathrm{J}=5.2 \mathrm{~Hz}, \mathrm{C}_{6}, \mathrm{~A}\right)$

MS (EI): 242, $240\left(\mathrm{M}^{+}\right)$ 
<smiles>[3H][Si](C)(C)C(=C(Br)C(F)(F)F)c1ccccc1</smiles>

${ }^{1} \mathrm{H}$ NMR $\left(300 \mathrm{MHz}, \mathrm{CDCl}_{3}\right)$ :

7.3-7.0 (m, 3H), 7.0-6.8 (m, 2H), 0.1-0 (s, 3H, two isomers)

${ }^{19} \mathrm{~F}$ NMR $\left(300 \mathrm{MHz}, \mathrm{CDCl}_{3}\right)$ :

$-39.61(\mathrm{~A}),-39.64(\mathrm{~B}), \mathrm{A} / \mathrm{B}=1 / 2.25$

${ }^{13} \mathrm{C} \mathrm{NMR}\left(75.5 \mathrm{MHz}, \mathrm{CDCl}_{3}\right)$ :

139.8 (d, J=11.3Hz, C3, B), 138.6 (C3, A), 138.2 (d, J=315, C1, B), 136.3 (d, J=331.8Hz, C1, A), 129.3 (C4, B), 129.1 (C4, A), 128.9 (d, J=3.2Hz, C5, B), 128.5 (d, J=1.4Hz, C5, A), 127.4 (C6, B), 127.3 (C6, A), 124.8 (d, J=27.6Hz, C2, B), $122.4(\mathrm{~d}, \mathrm{~J}=9.2 \mathrm{~Hz}, \mathrm{C} 2, \mathrm{~A}), 0.0$ (d, $\mathrm{J}=2.5 \mathrm{~Hz}, \mathrm{C} 7, \mathrm{~B}),-0.1(\mathrm{~d} ; \mathrm{J}=2.3 \mathrm{~Hz}, \mathrm{C} 7, \mathrm{~A})$

MS (EI): 274, $272\left(\mathrm{M}^{+}\right), 259,257\left(\mathrm{M}^{+}-\mathrm{CH}_{3}\right)$

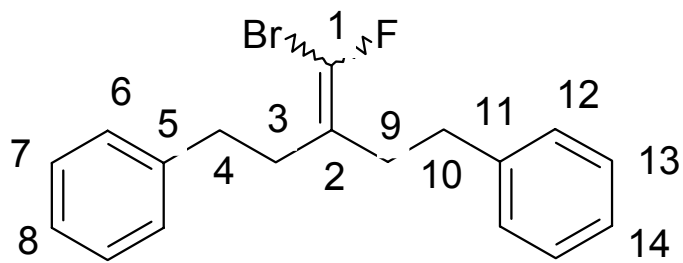

${ }^{1} \mathrm{H}$ NMR $\left(300 \mathrm{MHz}, \mathrm{CDCl}_{3}\right)$ :

7.4-7.0 (m, 10H), 2.8-2.6 (m, 4H), 2.6-2.4 (m, 2H), 2.4-2.2 (m, 2H)

${ }^{19} \mathrm{~F}$ NMR (282.5 $\left.\mathrm{MHz}^{\mathrm{CCDCl}} 3\right)$ :

$-77.6$

${ }^{13} \mathrm{C}$ NMR (75.5MHz, $\left.\mathrm{CDCl}_{3}\right)$ :

141.5-141.4 $\left(\mathrm{C}_{5}, \mathrm{C}_{11}\right), 131.6\left(\mathrm{~d}, \mathrm{~J}=312.8 \mathrm{~Hz}, \mathrm{C}_{1}\right), 128.9\left(\mathrm{C}_{7}, \mathrm{C}_{13}\right), 128.8-128.7\left(\mathrm{C}_{6}, \mathrm{C}_{12}\right), 126.6$ $\left(\mathrm{C}_{8}, \mathrm{C}_{14}\right), 120.5\left(\mathrm{~d}, \mathrm{~J}=10.4 \mathrm{~Hz}, \mathrm{C}_{2}\right), 34.7\left(\mathrm{C}_{4}\right), 34.5\left(\mathrm{~d}, \mathrm{~J}=6.8 \mathrm{~Hz}, \mathrm{C}_{9}\right), 33.9\left(\mathrm{~d}, \mathrm{~J}=4.0 \mathrm{~Hz}, \mathrm{C}_{10}\right)$, $31.3\left(\mathrm{~d}, \mathrm{~J}=4.6 \mathrm{~Hz}, \mathrm{C}_{3}\right)$ 
<smiles>CC(=CC=Cc1ccccc1)C(C)=C(C)O</smiles>

${ }^{1} \mathrm{H}$ NMR (300 MHz, $\left.\mathrm{CDCl}_{3}\right)$ :

7.5-7.2 (m, 5H), 7.2-7.1 (d, J=16Hz, 0.5H), 7.0-6.8 (d, J=16Hz, 0.5H), 2.0-1.8 (m, 3H, H9)

${ }^{19} \mathrm{~F}$ NMR (282.5 MHz, $\left.\mathrm{CDCl}_{3}\right)$ :

$-72.5(\mathrm{~d}, \mathrm{~J}=3.8 \mathrm{~Hz}, \mathrm{~A}),-74.4(\mathrm{~d}, \mathrm{~J}=4.3 \mathrm{~Hz}, \mathrm{~B}), \mathrm{A} / \mathrm{B}=1.00 / 1.23$

${ }^{13} \mathrm{C} \mathrm{NMR}\left(75.5 \mathrm{MHz}, \mathrm{CDCl}_{3}\right)$ :

$137.4\left(\mathrm{C}_{5}\right), 135.1$ (d, J=349.0Hz, $\left.\mathrm{C}_{1}, \mathrm{~A}\right), 135.0$ (d, J=350.7 Hz, $\left.\mathrm{C}_{1}, \mathrm{~B}\right), 131.4(\mathrm{~d}, \mathrm{~J}=12.1 \mathrm{~Hz}$, $\left.\mathrm{C}_{4}, \mathrm{~A}\right), 129.8\left(\mathrm{~d}, \mathrm{~J}=4.6 \mathrm{~Hz}, \mathrm{C}_{4}, \mathrm{~B}\right), 129.1\left(\mathrm{C}_{7}\right), 128.3\left(\mathrm{C}_{8}\right), 127.0\left(\mathrm{C}_{6}, \mathrm{~B}\right), 126.9(\mathrm{~d}, \mathrm{~J}=0.9 \mathrm{~Hz}$, $\left.\mathrm{C}_{6}, \mathrm{~A}\right), 126.1$ (d, J=0.9Hz, $\left.\mathrm{C}_{3}, \mathrm{~A}\right), 121.8$ (d, J=6.4Hz, $\left.\mathrm{C}_{3}, \mathrm{~B}\right), 117.4-117.0$ (dd, $\left.\mathrm{C}_{2}, \mathrm{~A}, \mathrm{~B}\right), 15.5$ $\left(\mathrm{C}_{9}, \mathrm{~B}\right), 12.0\left(\mathrm{C}_{9}, \mathrm{~A}\right)$

MS (EI):

242, $240\left(\mathrm{M}^{+}\right), 161\left(\mathrm{M}^{+}-\mathrm{Br}\right)$

IR $(\mathrm{KBr})$ :

3048, 3024, 2926, 1802, 1763, 1701, 1633, 1616, 1495, 1447, 1281, 1116, 1030, 958, 749, 691<smiles>FC(Br)=C1CCN(Cc2ccccc2)CC1</smiles>

${ }^{1} \mathrm{H}$ NMR $\left(300 \mathrm{MHz}, \mathrm{CDCl}_{3}\right)$ :

7.3-7.1 (m, 5H), 3.4 (s, 2H, H7), 2.5-2.3 (m, 6H), 2.3-2.1 (m, 2H)

${ }^{19}$ F NMR (282.5 $\left.\mathrm{MHz}^{\mathrm{CDCl}} \mathrm{CDC}_{3}\right)$ :

$-82.1$

${ }^{13} \mathrm{C} \mathrm{NMR}\left(75.5 \mathrm{MHz}, \mathrm{CDCl}_{3}\right)$ :

$138.5\left(\mathrm{C}_{8}\right), 129.5-128.7\left(\mathrm{C}_{9}, \mathrm{C}_{10}\right), 127.5\left(\mathrm{C}_{11}\right), 127.6-128.7\left(\mathrm{~d}, \mathrm{~J}=309.9 \mathrm{~Hz}, \mathrm{C}_{1}\right), 117.8(\mathrm{~d}$, $\left.\mathrm{J}=12.1 \mathrm{~Hz}, \mathrm{C}_{2}\right), 63.2\left(\mathrm{C}_{7}\right), 53.7-53.4\left(\mathrm{dd}, \mathrm{J}=1.7 \mathrm{~Hz}, \mathrm{~J}=2.3 \mathrm{~Hz}, \mathrm{C}_{4}, \mathrm{C}_{5}\right), 30.5\left(\mathrm{C}_{3}\right), 27.2(\mathrm{~d}$, $\mathrm{J}=5.2 \mathrm{~Hz}, \mathrm{C}_{6}$ )

MS (EI): 285, $283\left(\mathrm{M}^{+}\right), 204\left(\mathrm{M}^{+}-\mathrm{Br}\right)$ 


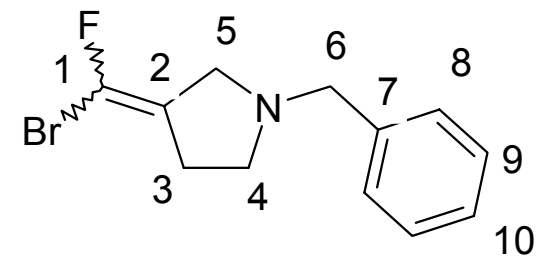

${ }^{1} \mathrm{H}$ NMR (300 MHz, $\left.\mathrm{CDCl}_{3}\right)$ :

7.5-7.2 (m, 5H), 3.8-3.6 (2s, 2H, H6), 3.4-3.3 (m, 1H, H5), 3.2-3.1 (m, 1H, H5), 2.9-2.7 (m, $2 \mathrm{H}, \mathrm{H} 4), 2.7-2.5(\mathrm{~m}, 1 \mathrm{H}, \mathrm{H} 3), 2.5-2.3(1 \mathrm{H}, \mathrm{H} 3)$

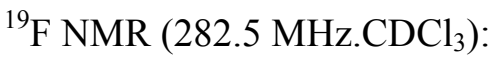

$-77.2(\mathrm{~A})),-79.4(\mathrm{~B}), \mathrm{A} / \mathrm{B}=1 / 1.26$

${ }^{13} \mathrm{C}$ NMR $\left(75.5 \mathrm{MHz}, \mathrm{CDCl}_{3}\right)$ :

$136.7\left(\mathrm{C}_{7}\right), 127.84,127.81,127.4,126.4\left(\mathrm{C}_{8}, \mathrm{C}_{9}, \mathrm{C}_{10}, \mathrm{~A}, \mathrm{~B}\right), 124.6\left(\mathrm{~d}, \mathrm{~J}=312.2 \mathrm{~Hz}, \mathrm{C}_{1}, \mathrm{~A}\right)$, $123.8\left(\mathrm{~d}, \mathrm{~J}=312.2 \mathrm{~Hz}, \mathrm{C}_{1}, \mathrm{~B}\right), 119.3\left(\mathrm{~d}, \mathrm{~J}=5.7 \mathrm{~Hz}, \mathrm{C}_{2}, \mathrm{~B}\right), 119.1\left(\mathrm{~d}, \mathrm{~J}=3.5 \mathrm{~Hz}, \mathrm{C}_{2}, \mathrm{~A}\right), 59.1-58.9$ $\left(\mathrm{C}_{6}, \mathrm{~A}, \mathrm{~B}\right), 56.0\left(\mathrm{~d}, \mathrm{~J}=2.3 \mathrm{~Hz}, \mathrm{C}_{5}, \mathrm{~B}\right), 54.0\left(\mathrm{~d}, \mathrm{~J}=8.9 \mathrm{~Hz}, \mathrm{C}_{5}, \mathrm{~A}\right), 53.4\left(\mathrm{C}_{4}, \mathrm{~B}\right), 52.3\left(\mathrm{C}_{4}, \mathrm{~A}\right), 29.0$ $\left(\mathrm{C}_{3}, \mathrm{~A}\right), 27.1\left(\mathrm{C}_{3}, \mathrm{~B}\right)$

MS (EI): 271, $269\left(\mathrm{M}^{+}\right), 180,178\left(\mathrm{M}^{+}-\mathrm{CH}_{2} \mathrm{Ph}\right)$ 